\title{
Research Article \\ The Effects of Chewing Gum in Preventing Eyestrain
}

\author{
Ken Asakawa $\mathbb{D}^{1},{ }^{1}$ Susumu Kanno, ${ }^{2}$ Tomonori Ando, ${ }^{2}$ Kenji Osawa, ${ }^{2}$ and Hitoshi Ishikawa ${ }^{1}$ \\ ${ }^{1}$ Department of Orthoptics and Visual Science, School of Allied Health Sciences, Kitasato University, Kanagawa, Japan \\ ${ }^{2}$ LOTTE Co., Ltd, Central Laboratory, Saitama, Japan \\ Correspondence should be addressed to Ken Asakawa; asaken@kitasato-u.ac.jp
}

Received 27 May 2020; Revised 22 October 2020; Accepted 27 October 2020; Published 16 November 2020

Academic Editor: Shigehiko Ogoh

Copyright (C) 2020 Ken Asakawa et al. This is an open access article distributed under the Creative Commons Attribution License, which permits unrestricted use, distribution, and reproduction in any medium, provided the original work is properly cited.

Purpose. To investigate the effects of chewing gum and tablet candy to reduce eyestrain in healthy individuals. Materials and Methods. A double-blinded crossover trial was conducted. Forty-six healthy individuals ( 23 men, 23 women) between 20 and 59 years old, feeling eyestrain, were enrolled. Each 10-year age group included 12 individuals except the 30s group, which included 10 individuals. A visual task was performed on reading material displayed on a computer screen at a fixed distance for 60 min. Gum or tablet candy of two pieces were chewed for two 15-min periods starting 15 and 45 min after starting to read. Subjects chewed gum on Day 1 and tablet candy on Day 2, and vice versa. Primary outcome is as follows: subjective eye fatigue (eye tiredness, eye heaviness, blurred vision, double vision, and eye dryness) using a visual analog scale (VAS). Secondary outcomes are as follows: subjective accommodation from near and far points of accommodation measured with a D'ACOMO, spherical equivalent refraction, and eye dryness by analyzing ring break-up time (RBUT) measured with the RT-7000 Auto RefTopographer. Results. The VAS scores of subjective eye fatigue were not significantly changed between chewing gum and tablet candy $(P=0.397-P=0.909)$. Those scores of eye tiredness and eye heaviness were significantly longer before and after the visual task with tablet candy $(P=0.013$ and $P=0.025$, respectively) but not with chewing gum. The changes of subjective accommodation were significantly lower after the visual task between chewing gum and candy $(P=0.043)$. There were significant differences among each age group $(20 \mathrm{~s}$ vs. $30 \mathrm{~s}, P=0.594 ; 20 \mathrm{~s}$ vs. $40 \mathrm{~s}, P=0.002 ; 20 \mathrm{~s}$ vs. $50 \mathrm{~s}, P=0.002)$. After reading, the changes of spherical equivalent refraction did not indicate a shift toward myopia $(P=0.267)$. In the RBUT, there were no significant differences between the samples $(P=0.680)$. Conclusions. Chewing gum helps improve the ability of the eye to focus, especially in young adults.

\section{Introduction}

Advances in information technology have encouraged the widespread adoption of new electronic devices, starting with visual display terminals (VDT), then notebook computers, and mobile phones, with email functions, and most recently, smartphones, and tablets. This has created a new visual environment (e.g., Information and Communication Technology; Internet of Things) and previously unknown formats for the input and output of visual information. As a result of this transformation of the environment, accommodative insufficiency after excessive close VDT work leads to subjective symptoms of blurry vision and difficulty focusing, causing multiple instances of eyestrain. The number of people complaining of these symptoms is increasing, and methods of combatting this issue must be found [1-4]. Resolution of these problems is therefore desirable.
Although many aspects of the underlying mechanism of eyestrain remain unclear, continuous near vision places the ciliary muscles of the eye under excessive strain, reducing the ability of the eye to focus (i.e., affecting accommodative function) [5-8]. Relaxing the ciliary muscles may thus help to prevent or improve eyestrain $[7,9]$. The best-known technique for this purpose is the use of a warm eye mask, which has been reported to increase blood flow to the ciliary muscles and improve the accommodative function [7]. The ciliary muscles, especially the circular Müller's fibers, which are innervated by the parasympathetic nerves, play an accommodative function.

Chewing is known to increase cerebral blood flow [10] and also actions on the autonomic nervous system (sympathetic and parasympathetic nerves) [11-13]. Our previous study showed that chewing gum increases blood flow to the 
eyes and also the parasympathetic nerves predominantly act to contract the iris sphincter muscle and thus reduce the diameter of the pupil [14]. The pupil is a small hole surrounded by the iris which controls its aperture by two smooth muscles, the iris dilator muscle (innervation by the sympathetic nerve) and the iris sphincter muscle (innervation by the parasympathetic nerve). Moreover, when viewing nearby objects, "near responses," consisting of convergence, accommodation, and pupil miosis are activated, and these responses are known to be cross-linked in the cortical center [15].

In this present study, we hypothesized that chewing gum may also activate the circular Müller's fibers of the ciliary muscle, which are innervated by the parasympathetic nerves, to improve the ability of the eye to focus (accommodative function) and thus reduce the subjective eye fatigue. A warm eye mask could not do so during VDT work; however, by chewing gum, this could possibly be accomplished. Thus, we considered that chewing gum could be a simple and easy way to prevent eyestrain. We, therefore, investigated the effects of chewing gum and tablet candy to reduce eyestrain in healthy individuals who felt eyestrain while using a computer or smartphone for prolonged periods.

\section{Materials and Methods}

2.1. Subjects. The subjects were recruited by a contract research organization, SOUKEN CO., Ltd. (Tokyo, Japan). They received a full explanation of the study and provided written informed consent. They were asked to refrain from drinking alcohol from the day before and throughout the study and were asked not to read printed materials, other than the test matter, and not watch videos on a smartphone or other device on the day of the study.

Exclusion criteria included pregnancy or lactation, taking regular dietary supplements, allergy to foods containing gelatin (e.g., gum and tablet candy), sufficiently severe dry eye such that refraining from blinking for 5 seconds was impossible, or an inability to either chew gum or tablet candy. Thus, 4 individuals ( 2 men, 2 women) who had severe dry eyes were excluded from the 30 s group, which had consisted of 14 individuals.

The study subjects were comprised of 46 individuals (23 men, 23 women) between 20 and 59 years old who felt eyestrain while using a computer or smartphone and who did not suffer from any eye disorders other than refractive abnormality. Each age group included 12 subjects ( 6 men, 6 women) except the $30 \mathrm{~s}$ group ( 5 men, 5 women); the mean ages for each group were $22.3 \pm 2.7$ (mean \pm standard deviation), $35.5 \pm 2.8,43.8 \pm 2.4$, and $55.4 \pm 3.0$ years for subjects in the 20 s, 30 s, 40 s, and 50 s groups, respectively.

2.2. Study Design. This was a double-blinded crossover trial. The subjects were divided into blocks by age and sex, and stratified randomization was conducted to allocate them to undergo measurements either by first chewing the test gum followed by chewing the control tablet candy or in the opposite order. There was no carryover effect evidenced with the testing (Table 1).
This study was approved by the Research Ethics Committee of Kitasato University School of Allied Health Sciences (approval No. 2019-001). This study was registered with the UMIN clinical trials registry (UMIN000037406).

2.3. Blinding of Subjects. Although the actual purpose of this study was to investigate the effect of chewing gum while viewing a computer screen, subjects were told that the purpose was to investigate the effect of sample substance (gum or tablet candy) while working on a computer. The subjects did not know which type of test substance they were given. The actual purpose of the study and the reason why it could not be disclosed in advance were explained at the end of the study.

2.4. Blinding of Examiners. Before starting the visual task, an examiner (S. Kanno) handed the samples, wrapped up to conceal the contents. The subjects were instructed to open the package and immediately put the contents into their mouths and start chewing the sample. During the visual reading task, the other examiner ( $K$. Asakawa) waited in a separate room from where the subjects could not be seen.

2.5. Testing. The tests were performed between 9:00 A.M. and 4:00 P.M. and were conducted in an examination room with a light level of approximately $500 \mathrm{~lx}$, a constant temperature of $24^{\circ} \mathrm{C}$, and humidity within the range of $40 \%-60 \%$. Tests following the procedures described in (1)-(6) below were carried out on 2 days, then evaluated. The starting times, food, and beverage intake (water ad libitum), for each participant, were matched on 2 days.

Primary outcome of testing is the following: subjective eye fatigue. Secondary outcomes of testing are the following: subjective accommodation, spherical equivalent refraction, and eye dryness. The tests were performed in the same order for each subject for each eye, right eye first (except the subjective eye fatigue).

(1) After arriving at the examination room, the subjects received an explanation of the study and provided written informed consent. They underwent routine visual acuity and refraction tests

(2) A 30-min break was provided

(3) Baseline subjective eye fatigue, subjective accommodation, spherical equivalent refraction, and eye dryness were measured

(4) A visual task was performed, comprised of reading on a computer screen at a fixed distance for $60 \mathrm{~min}$ (with a 1-min break after the first $30 \mathrm{~min}$ ). Subjects selected their own reading material from the free website (https://www.satokazzz.com/books/)

(5) A sample substance (gum or tablet candy of two pieces) was chewed for two 15-min periods starting at 15 and $45 \mathrm{~min}$ after starting reading. Subjects who chewed gum on Day 1 chewed tablet candy on Day 2, and vice versa 
TABLE 1: Reproducibility of the values before chewing gum or tablet candy (carryover effect).

\begin{tabular}{|c|c|c|c|c|c|c|}
\hline \multirow[b]{2}{*}{ Outcomes } & \multirow[b]{2}{*}{ Number of data } & \multirow[t]{2}{*}{ Difference of mean value before chewing sample } & \multirow[b]{2}{*}{ SD } & \multicolumn{2}{|c|}{$\begin{array}{l}\text { 95\% confidence } \\
\text { interval }\end{array}$} & \multirow[b]{2}{*}{$P$ value } \\
\hline & & & & $\begin{array}{l}\text { Lower } \\
\text { limit }\end{array}$ & $\begin{array}{l}\text { Upper } \\
\text { limit }\end{array}$ & \\
\hline Subjective accommodation (D) & & -0.03 & 0.97 & -0.23 & 0.17 & 0.786 \\
\hline Spherical equivalent refraction (D) & 92 & -0.03 & 0.29 & -0.09 & 0.03 & 0.393 \\
\hline Ring break-up time (sec) & & -0.04 & 3.31 & -0.73 & 0.64 & 0.903 \\
\hline
\end{tabular}

SD: standard deviation. Paired $t$-test.

(6) Subjective eye fatigue, subjective accommodation, spherical equivalent refraction, and eye dryness were measured after the visual reading task

2.6. Sample Substance. Gum (1.0 g/piece) contained maltitol, gum base, mint flavor, gelatin, sugar ester, and glycerin. Tablet candy $(0.7 \mathrm{~g} / \mathrm{piece})$ contained the same nutritional ingredients and flavor as the gum except for the gum base. The amount of energy and the constitution of the gum and the tablet candy were the same (carbohydrate, protein, and fat: 100,0 , and 0 , respectively).

\subsection{Primary Outcome of Testing}

2.7.1. Subjective Eye Fatigue. Five categories were evaluated using a visual analog scale (VAS): eye tiredness, eye heaviness, blurred vision, double vision, and eye dryness. The severity of the complaint was evaluated on a scale with $0 \mathrm{~mm}$ representing "not present at all" and $100 \mathrm{~mm}$ as "very severe." Medical questionnaires were not used in this investigation of subjective symptoms to avoid asking leading questions, and the subjects indicated their current conditions themselves by drawing a perpendicular line across the scale for each of the five categories.

\subsection{Secondary Outcomes of Testing}

2.8.1. Subjective Accommodation. This value was measured with a D'ACOMO (WOC, Kyoto, Japan). A visual target (yellow cross) was moved toward the subject at $0.2 \mathrm{D} / \mathrm{s}$ to determine the accommodation near point and away from them to determine the far point of accommodation. Distances at which the target became blurred were measured three times each (in centimeters), and amplitude of subjective accommodation (D) was calculated from the values of the near and far points of accommodation. The measurements were performed wearing $\mathrm{a}+4.0 \mathrm{D}$ lens when measuring far points and when subjects could only see the blurred visual target. Subjective accommodation was then calculated as 100/near point - 100/far point.

2.8.2. Spherical Equivalent Refraction. Spherical and cylindrical powers were measured three times with the RT-7000 Auto Ref-Topographer (Tomey, Nagoya, Japan), and the spherical equivalent refraction diopter (D) was calculated from those values.

2.8.3. Eye Dryness. This value was measured with the RT7000 Auto Ref-Topographer and Tear Stability Analysis System (TSAS) (Tomey, Nagoya, Japan). The subject was instructed to keep the eyes open for $10 \mathrm{sec}$. Severity of eye dryness was then evaluated by measuring ring break-up time (RBUT). In accordance with the algorithm provided by Tomey, the RBUT was measured by analyzing break-up of the tear layer within a 6-mm radius of the center of the cornea and its deformation over time and measuring the number of seconds required to reach the cut-off value of $-0.5 \mathrm{D}$.

2.9. Statistical Analyses. Statistical analyses were performed by ESUMI Co., Ltd., (Tokyo, Japan) using SPSS, version 26.0 (IBM, Armonk, NY, USA). Carryover effect of the testing was confirmed using the Paired $t$-test. Results are presented as mean \pm standard deviations. Comparisons of between $(\Delta)$ the chewing gum and tablet candy and before and after the chewing gum or tablet candy were performed using the Wilcoxon signed-rank test. Effect size $(r)$ was also calculated. Each age group was compared using the Kruskal-Wallis test, and the Mann-Whitney $U$ test with Bonferroni's correction was used to determine the differences among each age group for post hoc comparisons. Results are presented as median and interquartile range (25th, 75th percentiles). $P$ values of $<0.05$ were considered statistically significant.

\section{Results}

Table 2 shows the data of between the chewing gum and tablet candy in all the subjects, and those in each age group are shown in Table 3. Table 4 shows the data of before and after the chewing gum or tablet candy in all the subjects.

\subsection{Primary Outcome of Testing}

3.1.1. Subjective Eye Fatigue. The VAS scores of subjective eye fatigue were not significantly changed after the visual task between chewing gum and tablet candy in any of the categories (eye tiredness, $P=0.657$; eye heaviness, $P$ $=0.694$; blurred vision, $P=0.909$; double vision, $P=$ 0.397 ; eye dryness, $P=0.757)$. There were no significant differences among any of the age groups with chewing gum and tablet candy. Compared to before the visual task, the VAS scores of eye tiredness and eye heaviness were significantly longer after the visual task with tablet candy ( $P=0.013$ and $P=0.025$, respectively) but not with chewing gum $(P=0.059$ and $P=0.075$, respectively).

\subsection{Secondary Outcomes of Testing}

3.2.1. Subjective Accommodation. The changes in subjective accommodation were significantly lower after the visual task 
TABLE 2: Data of between chewing gum and tablet candy in all subjects.

\begin{tabular}{|c|c|c|c|c|c|c|}
\hline Outcomes & & Number of data & \multicolumn{2}{|c|}{ Median $(\mathrm{IQR})$} & $P$ value & $r$ \\
\hline \multirow{8}{*}{ VAS $(\mathrm{mm})$} & Eye tiredness & & $1.5(-6.5,19.8)$ & $11.5(-7.8,28.5)$ & 0.657 & 0.065 \\
\hline & Eye heaviness & & $3.5(-9.3,19.5)$ & $4.5(-4.3,25.3)$ & 0.694 & 0.058 \\
\hline & Blurred vision & 46 & $0(-2.3,15.0)$ & $0(-5.8,19.3)$ & 0.909 & 0.017 \\
\hline & Double vision & & $0(-1.0,7.3)$ & $1.0(-1.3,14.5)$ & 0.397 & 0.125 \\
\hline & Eye dryness & & $0(-9.8,16.3)$ & $1.0(-12.0,19.3)$ & 0.757 & 0.046 \\
\hline & Subjective accommodation (D) & & $-0.04(-0.32,0.42)$ & $-0.14(-0.50,0.22)$ & $0.043^{*}$ & 0.211 \\
\hline & Spherical equivalent refraction (D) & 92 & $0(-0.25,-0.13)$ & $0(-0.25,0.13)$ & 0.267 & 0.116 \\
\hline & Ring break-up time (sec) & & $0(-2.35,0.15)$ & $0(-1.15,0)$ & 0.680 & 0.043 \\
\hline
\end{tabular}

IQR: interquartile range (25th, 75th percentiles); VAS: visual analog scale. ${ }^{*} P<0.05$; Wilcoxon signed-rank test. $r$ : effect size.

TABLE 3: Data of between chewing gum and tablet candy in each age group.

\begin{tabular}{|c|c|c|c|c|c|c|c|}
\hline & \multirow{2}{*}{\multicolumn{2}{|c|}{ Outcomes }} & $20 \mathrm{~s}(n=12)$ & $30 \mathrm{~s}(n=10)$ & $40 \mathrm{~s}(n=12)$ & $50 \mathrm{~s}(n=12)$ & \\
\hline & & & \multicolumn{5}{|c|}{ Median (IQR) } \\
\hline \multirow{8}{*}{$\operatorname{Gum}(\Delta)$} & \multirow{8}{*}{ VAS (mm) } & Eye tiredness & $7.0(-4.5,21.3)$ & $2.5(-5.5,46.3)$ & $0(-5.3,11.8)$ & $1.5(-15.3,23.8)$ & 0.794 \\
\hline & & Eye heaviness & $5.0(-9.3,19.5)$ & $9.5(-4.0,25.8)$ & $-0.5(-24.8,13.0)$ & $8.0(-8.3,27.3)$ & 0.424 \\
\hline & & Blurred vision & $0(-0.8,6.3)$ & $5.5(0,21.3)$ & $0(-15.8,20.5)$ & $-2.0(-11.5,11.8)$ & 0.537 \\
\hline & & Double vision & $0(-1.8,5.8)$ & $0.5(-0.3,16.3)$ & $0(0,8.8)$ & $0.5(-4.0,13.3)$ & 0.789 \\
\hline & & Eye dryness & $3.0(-11.3,12.8)$ & $17.5(-0.3,36.3)$ & $-1.5(-15.3,10.3)$ & $-0.5(-17.3,6.8)$ & 0.240 \\
\hline & & $\begin{array}{c}\text { Subjective } \\
\text { accommodation (D) }\end{array}$ & $-0.2(-0.9,0.4)$ & $-0.2(-0.6,0.2)$ & $0.2(-0.1,0.6)$ & $-0.1(-0.2,0.3)$ & 0.055 \\
\hline & & $\begin{array}{l}\text { Spherical equivalent } \\
\text { refraction (D) }\end{array}$ & $0(-0.1,0.2)$ & $0(-0.3,0)$ & $-0.1(-0.4,0.3)$ & $0(-0.3,0.1)$ & 0.345 \\
\hline & & $\begin{array}{l}\text { Ring break-up } \\
\text { time }(\mathrm{sec})\end{array}$ & $-0.1(-3.2,0)$ & $0(-4.2,0)$ & $-0.2(-2.8,2.2)$ & $0(-1.3,0.6)$ & 0.417 \\
\hline \multirow{8}{*}{$\begin{array}{l}\text { Tablet } \\
\text { candy }(\Delta)\end{array}$} & \multirow{8}{*}{ VAS (mm) } & Eye tiredness & $14.5(9.0,34.3)$ & $14.0(-17.5,27.5)$ & $0.5(-14.0,24.3)$ & $15.5(-14.5,34.8)$ & 0.524 \\
\hline & & Eye heaviness & $3.0(-0.8,35.0)$ & $4.0(-7.8,20.8)$ & $-1.0(-17.3,10.0)$ & $14.0(-1.8,28.5)$ & 0.355 \\
\hline & & Blurred vision & $2.0(-1.5,18.3)$ & $2.0(-20.3,31.0)$ & $2.5(-10.3,17.8)$ & $-1.5(-9.5,18.5)$ & 0.792 \\
\hline & & Double vision & $0.5(-3.3,9.0)$ & $0(-1.8,20.5)$ & $4.0(0,19.5)$ & $5.5(-2.0,12.0)$ & 0.514 \\
\hline & & Eye dryness & $11.0(-0.8,32.5)$ & $5.5(-8.3,37.0)$ & $0(-15.8,10.0)$ & $0(-25.5,17.5)$ & 0.237 \\
\hline & & $\begin{array}{c}\text { Subjective } \\
\text { accommodation (D) }\end{array}$ & $-0.7(-1.4,-0.2)$ & $-0.2(-0.9,0.3)$ & $0.1(-0.1,0.4)^{\dagger}$ & $0(-0.2,0.2)^{\ddagger}$ & $<0.001^{* * *}$ \\
\hline & & $\begin{array}{l}\text { Spherical equivalent } \\
\text { refraction (D) }\end{array}$ & $0(-0.3,0.1)$ & $0(0,0.1)$ & $-0.3(-0.4,0)^{\S}$ & $0(-0.3,0.1)$ & $0.010^{* *}$ \\
\hline & & Ring break-up time (sec) & $0(-1.7,0)$ & $0(-5.9,0)$ & $0(-0.9,0)$ & $0(-0.1,1.4)$ & 0.221 \\
\hline
\end{tabular}

IQR: interquartile range (25th, 75th percentiles); VAS: visual analog scale. ${ }^{* *} P<0.01,{ }^{* * *} P<0.001$; Kruskal-Wallis test. ${ }^{\dagger} P=0.002, r=0.527(20 \mathrm{~s}$ vs. $40 \mathrm{~s}$ ), ${ }^{\ddagger} P=0.002, r=0.513$ (20 s vs. $50 \mathrm{~s}$ ), ${ }^{\S} P=0.007, r=0.487$ (30 s vs. $40 \mathrm{~s}$ ); Mann-Whitney $U$ test with Bonferroni's correction. $r$ : effect size.

between the chewing gum and tablet candy $(P=0.043, r=$ 0.211). There were significant differences among each age group $(P<0.001)$ (20 s vs. $30 \mathrm{~s}, P=0.594, r=0.249 ; 20 \mathrm{~s}$ vs. 40 s, $P=0.002, r=0.527 ; 20$ s vs. 50 s, $P=0.002, r=0.513)$. This median value was $3.9 \mathrm{D}$ and $4.0 \mathrm{D}$ with chewing gum and $4.2 \mathrm{D}$ and $3.8 \mathrm{D}$ with tablet candy before and after the visual reading task, respectively (chewing gum, $P=0.579$; tablet candy, $P=0.014$ ).

3.2.2. Spherical Equivalent Refraction. The changes of spherical equivalent refraction did not indicate a shift toward myopia $(P=0.267)$. Significant differences were obtained in each age group $(P=0.010)$. There were significant differences between the $30 \mathrm{~s}$ and $40 \mathrm{~s}$ groups $(P=0.007, r=0.487)$. The values of before and after the visual task were $-1.19 \mathrm{D}$ and $-1.00 \mathrm{D}$, respectively, for chewing gum $(P=0.361)$ and $-1.00 \mathrm{D}$ and $-1.25 \mathrm{D}$, respectively, for chewing tablet candy $(P=0.008)$.

3.2.3. Eye Dryness. There were no significant differences between chewing gum and tablet candy $(P=0.680)$. Before and after the visual task, the RBUT values were $10.0 \mathrm{sec}$ and $9.6 \mathrm{sec}$, respectively, with chewing gum $(P=0.053)$ and $9.9 \mathrm{sec}$ and $9.6 \mathrm{sec}$, respectively, with tablet candy $(P=0.132)$. 
TABle 4: Data of before and after chewing gum or tablet candy in all subjects.

\begin{tabular}{|c|c|c|c|c|c|c|c|}
\hline Sample & \multicolumn{2}{|r|}{ Outcomes } & \multirow[t]{2}{*}{ Number of data } & \multicolumn{2}{|c|}{ Median (IQR) } & \multirow{2}{*}{$\frac{P \text { value }}{0.059}$} & \multirow{2}{*}{$\begin{array}{c}r \\
0.278\end{array}$} \\
\hline \multirow{8}{*}{ Gum } & \multirow{8}{*}{ VAS (mm) } & Eye tiredness & & $29(4,61)$ & $45(20,66)$ & & \\
\hline & & Eye heaviness & & $15(2,42)$ & $28(8,50)$ & 0.075 & 0.263 \\
\hline & & Blurred vision & 46 & $15(1,55)$ & $21(1,54)$ & 0.155 & 0.210 \\
\hline & & Double vision & & $4(0,20)$ & $9(1,44)$ & $0.032^{*}$ & 0.316 \\
\hline & & Eye dryness & & $25(2,48)$ & $32(8,54)$ & 0.401 & 0.124 \\
\hline & & Subjective accommodation (D) & & $3.9(2.7,6.4)$ & $4.0(2.7,6.4)$ & 0.579 & 0.058 \\
\hline & & Spherical equivalent refraction (D) & 92 & $-1.19(-4.09,-0.25)$ & $-1.00(-4.34,-0.25)$ & 0.361 & 0.095 \\
\hline & & Ring break-up time (sec) & & $10.0(4.7,10.0)$ & $9.6(3.4,10.0)$ & 0.053 & 0.202 \\
\hline \multirow{8}{*}{ Tablet candy } & \multirow{8}{*}{ VAS $(\mathrm{mm})$} & Eye tiredness & & $24(4,61)$ & $45(22,63)$ & $0.013^{*}$ & 0.366 \\
\hline & & Eye heaviness & & $17(4,47)$ & $32(9,58)$ & $0.025^{*}$ & 0.330 \\
\hline & & Blurred vision & 46 & $12(1,46)$ & $29(4,47)$ & 0.159 & 0.208 \\
\hline & & Double vision & & $3(1,20)$ & $12(1,34)$ & $0.009^{* *}$ & 0.384 \\
\hline & & Eye dryness & & $28(7,58)$ & $42(4,54)$ & 0.237 & 0.174 \\
\hline & & Subjective accommodation (D) & & $4.2(2.7,6.3)$ & $3.8(2.5,6.0)$ & $0.014^{*}$ & 0.256 \\
\hline & & Spherical equivalent refraction (D) & 92 & $-1.00(-3.97,-0.38)$ & $-1.25(-4.34,-0.38)$ & $0.008^{* *}$ & 0.277 \\
\hline & & Ring break-up time (sec) & & $9.9(5.3,10.0)$ & $9.6(4.3,10.0)$ & 0.132 & 0.157 \\
\hline
\end{tabular}

IQR: interquartile range (25th, 75th percentiles); VAS: visual analog scale. ${ }^{*} P<0.05,{ }^{* *} P<0.01$; Wilcoxon signed-rank test. $r$ : effect size.

\section{Discussion}

The effects of chewing gum demonstrated that the maintenance of homeostasis, in the forms of blood pressure and heart rate, was mediated by the sympathetic and parasympathetic nerves [11-13]. Actions on the autonomic nervous system of the eye were also reported [14]. In a previous study, we found that pupil diameter contracted significantly after chewing gum compared with that at the baseline before chewing gum in young adults [14]. Whether that was due to sympathetic nerve inhibition or parasympathetic nerve activity had been unclear; however, we concluded that it was most likely due to the predominating parasympathetic nerve activity. Furthermore, previous studies have demonstrated that mastication affects human cognitive processing, including choice reaction time [16], positive mood [17], working memory $[18,19]$, somatosensory processing [20], motor preparation [21], and Go/No-go decision-making [22].

One factor involved in the mechanism of eye fatigue is central fatigue due to processing visual information associated with cognitive function. Subjective fatigue reflects fatigue in the cognitive processes in the central nervous system. A study of cerebral blood flow responses while fatigued found that the blood flow response decreased in associated areas of the visual cortex [23]. Conversely, the increased cerebral blood flow, as a result of chewing gum, has been suggested to help increase alertness or at least attenuate reductions in alertness [24, 25]. Regarding the findings related to relaxation caused by odor and/or taste, rather than mastication, we used samples with the same odors and tastes. Moreover, because the ability to concentrate on a visual task could be decreased during chewing gum, thereby possibly decreasing eyestrain, we checked the number of words each participant read during the chewing gum and tablet candy trials. There were no significant differ- ences in the number of words read between the gum and tablet candy trials. We therefore considered that the gum and candy trials were carried out in as nearly the same conditions as possible, except for mastication. On the other hand, the effects of mastication may have differed among the subjects. These effects may be related to the frequency of chewing gum in daily life. There may be a relationship between the typical use of chewing gum and the changes in this study. We therefore found that there is no difference in the subjective eye fatigue between the gum and candy trials. However, before and after chewing gum, eye tiredness and eye heaviness was reduced. It is warranted to investigate this issue with subjects whose lifestyle habits, e.g., frequency of chewing gum, are the same. This could be considered a limitation in this study.

Eye fatigue is also believed to be caused by the ciliary muscles, which are involved in the accommodative function [5-8]; and warming the area around the eyes has been shown to increase blood flow and improve the accommodative function [9]. In our previous findings, chewing gum increased blood flow to the area around the eyes and caused the parasympathetic nerves predominantly act to contract the pupillary sphincter [14]. Our findings in the present study showed that chewing gum significantly reduced declines in the amplitude of subjective accommodation after the visual reading task, which may have been due to factors including improved blood flow in the eyes and activation of the Müller's ciliary muscles by the parasympathetic nerves. The clinical implication has less significance for affecting accommodative function from a little bit of effect size. However, there were significant differences among each age group, especially the $20 \mathrm{~s}$ group. Therefore, chewing gum helps to inhibit the declines in subjective accommodation and to improve the ability of the eye to focus by the action of the parasympathetic nerves in young adults who have sufficient accommodative function. 
A previous study found that if the ciliary muscles remain in a continuous state of strain due to VDT work, transient myopia develops [26]. However, there were no significant changes in the spherical equivalent refraction after the visual task and no shift toward myopia (only $0.25 \mathrm{D}$ ) in the present study. Thus, fluctuations in accommodation may have been involved. "Fluctuations in accommodation" refers to the fact that even when the eye is focused on a stationary target, its refraction is not constant but exhibits slight fluctuations [27]. Because the value is around $0.3 \mathrm{D}$, the change after the visual reading task when subjects were chewing gum or tablet candy was only a slight change that was not reflected in subjective symptoms (e.g., blurry vision and difficulty focusing).

Factors reported to affect dry eye include a reduced frequency of blinking during VDT work [28] and reduced tear secretion due to increased sympathetic nervous activity as a result of stress or other causes [29], and lacrimal gland hypofunction [30]. Because tear production by the lacrimal glands is stimulated by the parasympathetic nerves [31], if chewing gum results in parasympathetic nerves predominance, tear production may also be improved. However, in the present study, there were no significant differences between each sample substance in the values of RBUT with TSAS. Tears are also derived from the meibomian glands, and meibomian secretions help stabilize the tear film [32]. One of the positive aspects is that chewing gum may help activate the lacrimal glands but not the meibomian glands, and they could conceivably also help to alleviate eye dryness. However, it is still unclear how chewing gum involves the lacrimal glands.

\section{Conclusions}

These results show that in healthy individuals, especially in young adults, who feel eyestrain, chewing gum helps to inhibit the declines in subjective accommodation and to improve the ability of the eye to focus by the action of the parasympathetic nerves. However, the mechanisms underlying eye fatigue are extremely complex, and further in-depth investigations are warranted to elucidate the changes that take place in the eye.

\section{Data Availability}

The data used to support the findings of this study are included within the article.

\section{Conflicts of Interest}

Ken Asakawa received support for this study from LOTTE Co., Ltd. The other authors have no proprietary or commercial interest in any materials discussed in this article.

\section{Acknowledgments}

The authors thank Robert E. Brandt, Founder, CEO, and CME, of MedEd Japan, for editing and formatting the manuscript. LOTTE Co., Ltd., funded this study and provided the sample substances (gum and tablet candy) without fee.

\section{References}

[1] M. Rosenfield, P. A. Howarth, J. E. Sheedy, and M. D. Crossland, "Vision and IT displays: a whole new visual world," Ophthalmic and Physiological Optics, vol. 32, no. 5, pp. 363-366, 2012.

[2] J. Long, R. Cheung, S. Duong, R. Paynter, and L. Asper, "Viewing distance and eyestrain symptoms with prolonged viewing of smartphones," Clinical and Experimental Optometry, vol. 100, no. 2, pp. 133-137, 2017.

[3] E. Porcar, J. C. Montalt, Á. M. Pons, and E. España-Gregori, "Symptomatic accommodative and binocular dysfunctions from the use of flat-panel displays," International Journal of Ophthalmology, vol. 11, no. 3, pp. 501-505, 2018.

[4] B. Golebiowski, J. Long, K. Harrison, A. Lee, N. Chidi-Egboka, and L. Asper, "Smartphone use and effects on tear film, blinking and binocular vision," Current Eye Research, vol. 45, no. 4, pp. 428-434, 2020.

[5] W. Jaschinski-Kruza and W. Schweflinghaus, "Relations between dark accommodation and psychosomatic symptoms," Ophthalmic and Physiological Optics, vol. 12, no. 1, pp. 103105, 1992.

[6] J. M. Feldman, J. Cooper, F. Reinstein, and J. Swiatoca, “Asthenopia induced by computer-generated fusional vergence targets," Optometry and Vision Science, vol. 69, no. 9, pp. 710716, 1992.

[7] M. Kajita, M. Ono, S. Suzuki, and K. Kato, "Accommodative microfluctuation in asthenopia caused by accommodative spasm," Fukushima Journal of Medical Science, vol. 47, no. 1, pp. 13-20, 2001.

[8] H. M. Thorud, M. Helland, A. Aarås, T. M. Kvikstad, L. G. Lindberg, and G. Horgen, "Eye-related pain induced by visually demanding computer work," Optometry and Vision Science, vol. 89, no. 4, pp. 452-464, 2012.

[9] Y. Takahashi, M. Igaki, A. Suzuki, G. Takahashi, M. Dogru, and K. Tsubota, "The effect of periocular warming on accommodation," Ophthalmology, vol. 112, no. 6, pp. 1113-1118, 2005.

[10] T. Momose, J. Nishikawa, T. Watanabe et al., "Effect of mastication on regional cerebral blood flow in humans examined by positron-emission tomography with 15O-labelled water and magnetic resonance imaging," Archives of Oral Biology, vol. 42, no. 1, pp. 57-61, 1997.

[11] I. Ishiyama and M. Suzuki, "Blood pressure and cerebral blood flow volume responses to mastication of food," Journal of Japanese Society for Mastication Science and Health Promotion, vol. 15, no. 1, pp. 24-36, 2005.

[12] Y. Hasegawa, T. Ono, K. Hori, and T. Nokubi, "Influence of human jaw movement on cerebral blood flow," Journal of Dental Research, vol. 86, no. 1, pp. 64-68, 2007.

[13] S. Kanno, K. Shimo, T. Ando, Y. Hamada, M. Miyashita, and K. Osawa, "Gum chewing while walking increases fat oxidation and energy expenditure," Journal of Physical Therapy Science, vol. 31, no. 5, pp. 435-439, 2019.

[14] K. Asakawa, H. Ishikawa, N. Shoji, and T. Suzuki, "Effect of mastication on the autonomic nervous system in the human eye," The Autonomic Nervous System, vol. 44, no. 2, pp. 98 $103,2007$.

[15] K. Asakawa, M. Tomioka, J. Nakayama, M. Hayama, M. Hinata, and H. Ishikawa, "Crosslinking of near responses in healthy young subjects," Acta Ophthalmologica, vol. 98, no. 6 , pp. 791-793, 2020. 
[16] N. S. Chu, "Effect of betel chewing on performance reaction time," Journal of the Formosan Medical Association, vol. 93, no. 4, pp. 343-345, 1994.

[17] A. Smith, "Effects of caffeine in chewing gum on mood and attention," Human Psychopharmacology: Clinical and Experimental, vol. 24, no. 3, pp. 239-247, 2009.

[18] L. Wilkinson, A. Scholey, and K. Wesnes, "Chewing gum selectively improves aspects of memory in healthy volunteers," Appetite, vol. 38, no. 3, pp. 235-236, 2002.

[19] Y. Hirano, T. Obara, K. Kashikura et al., "Effects of chewing in working memory processing," Neuroscience Letters, vol. 436, no. 2, pp. 189-192, 2008.

[20] H. Nakata, M. Aoki, and K. Sakamoto, "Effects of mastication on human somatosensory processing: a study using somatosensory-evoked potentials," Neuroscience Research, vol. 117, pp. 28-34, 2017.

[21] K. Sakamoto, H. Nakata, Y. Honda, and R. Kakigi, “The effect of mastication on human motor preparation processing: a study with CNV and MRCP," Neuroscience Research, vol. 64, no. 3, pp. 259-266, 2009.

[22] K. Sakamoto, H. Nakata, M. Yumoto, N. Sadato, and R. Kakigi, "Mastication accelerates Go/No-go decisional processing: an event-related potential study," Clinical Neurophysiology, vol. 126, no. 11, pp. 2099-2107, 2015.

[23] M. Tanaka, N. Sadato, T. Okada et al., "Reduced responsiveness is an essential feature of chronic fatigue syndrome: a fMRI study," BMC Neurology, vol. 6, no. 1, article 9, 2006.

[24] Y. Hirano, T. Obata, H. Takahashi et al., "Effects of chewing on cognitive processing speed," Brain and Cognition, vol. 81, no. 3, pp. 376-381, 2013.

[25] A. P. Allen and A. P. Smith, "Chewing gum: cognitive performance, mood, well-being, and associated physiology," BioMed Research International, vol. 2015, Article ID 654806, 16 pages, 2015.

[26] T. Iwasaki, A. Tawara, and N. Miyake, "Reduction of asthenopia related to accommodative relaxation by means of far point stimuli," Acta Ophthalmologica Scandinavica, vol. 83, no. 1, pp. 81-88, 2005.

[27] W. N. Charman and G. Heron, "Microfluctuations in accommodation: an update on their characteristics and possible role," Ophthalmic and Physiological Optics, vol. 35, no. 5, pp. 476-499, 2015.

[28] K. Tsubota and K. Nakamori, "Dry eyes and video display terminals," The New England Journal of Medicine, vol. 328, no. 8, p. $584,1993$.

[29] M. Uchino, D. A. Schaumberg, M. Dogru et al., "Prevalence of dry eye disease among Japanese visual display terminal users," Ophthalmology, vol. 115, no. 11, pp. 1982-1988, 2008.

[30] S. Nakamura, S. Kinoshita, N. Yokoi et al., "Lacrimal hypofunction as a new mechanism of dry eye in visual display terminal users," PLoS One, vol. 5, no. 6, article e11119, 2010.

[31] D. A. Dartt, "Neural regulation of lacrimal gland secretory processes: relevance in dry eye diseases," Progress in Retinal and Eye Research, vol. 28, no. 3, pp. 155-177, 2009.

[32] R. Arita, S. Fukuoka, and N. Morishige, "New insights into the morphology and function of meibomian glands," Experimental Eye Research, vol. 163, pp. 64-71, 2017. 